\title{
Einsatz von RFID in der Pharmazeutischen Industrie
}

\author{
PROF. DR.-ING. R. JANSEN \\ DiPL. WIRT. INF. MARTIN HELMIGH \\ FACHGEBIET LOGISTIK (FLOG) DER FAKULTÄT MASCHINENBAU, UNIVERSITÄT DORTMUND
}

\section{Zusammenfassung}

Die Radio Frequenz Identifikation (RFID) gilt als wichtigste technologische Neuerung in der Informationslogistik. Wird RFID in der produzierenden Industrie hauptsächlich zur Betriebsdatenerfassung und im Handel zur Wareneingangs-/ Warenausgangskontrolle verwendet, so können insbesondere in der Pharmazeutischen Industrie die Vorteile der Technologie voll ausgereizt werden. Die wohl wichtigste Anwendung ist die lückenlose Rückverfolgung entlang der Lieferkette, wie sie in den USA bereits in einigen Staaten für alle pharmazeutischen Produkte vorgeschrieben und auch in Deutschland für bestimmte Produkte erforderlich ist. Zudem können die RFID Transponder auf der Produktverpackung als fälschungssicheres Merkmal eingesetzt werden. Bei temperatursensiblen Produkten können Transponder mit zusätzlicher Sensorik zur Überwachung der Kühlkette dienen. Gleichzeitig kann der Transponder auch als Diebstahlsicherung im innerbetrieblichen Bereich sowie auch im Handel dienen und ermöglicht dabei eine höhere Sicherheit als die bisher eingesetzten 1-Bit Transponder. Die Transpondertechnologie kann außerdem den Barcode ganz oder teilweise ersetzen und so einen großen Beitrag zur Prozessautomatisierung leisten.

\begin{abstract}
Radio frequency identification (RFID) is considered to be the most important technological innovation in information logistics. While RFID is mainly used for capturing production data in the producing industry and controlling goods issue/ goods received in commerce, the full advantage of the technology can be deployed in the pharmaceutical industry. The most important application is probably the complete tracking and tracing of goods along the entire supply chain, which is already required for all pharmaceutical products in some states in the US and also for certain products in Germany. In addition the RFID transponder can be used as seal of authenticity if it is applied to the packaging. Transponders with additional sensor technology can supervise the cooling chain of temperature sensitive products. Furthermore the transponder can also serve as an anti theft device as well within the company as in stores and enables a higher security level than the 1-Bit Transponder used so far. The transponder technology can also replace the bar code totally or partly and is therefore a large contribution for process automation.
\end{abstract}

\section{Fälschungssicherung durch Rückverfolgung}

Die Rückverfolgung und Quellensicherung sind ein probates Mittel, um die Qualität eines Produktes zu sichern und erschweren das Einbringen von gefälschten Produkten. Um eine Verfolgung über die gesamte Lieferkette zu gewährleisten, bedarf es nicht unbedingt eines flächendeckenden Einsatzes der RFID-Technologie. Ein entscheidender Faktor ist dabei der Integrationsgrad der Supply Chain; ist dieser bereits weit vorangeschritten, vereinfacht dies die Einführung von RFID. Die RFID-Software muss dann nur so in die Supply Chain Software eingepasst werden, dass Fertigungsrückmeldungen, Lagerortumbuchungen und Warenein-/ Warenausgangsbuchungen automatisiert erfolgen und so die Rückverfolgung ermöglichen. Durch den Einsatz von Smartlabeln können kleinere Supply Chain Partner sowie Teile der Lieferkette, für die der RFID-Einsatz ineffizient ist, weiterhin auf Barcode-Basis arbeiten. Zudem können die Investitionskosten für die Transponder unter den Supply ChainPartnern aufgeteilt werden. Wie bei allen Supply Chain-Projekten ist der Vertrauensgrad zwischen den Unternehmen ein wichtiger Faktor, da Informationen über Mengen, Prozess- und Lagerzeiten allen Partnern zugänglich sind. Außerdem muss geklärt sein, wie die Kosten unter den Partnern aufgeteilt werden. 


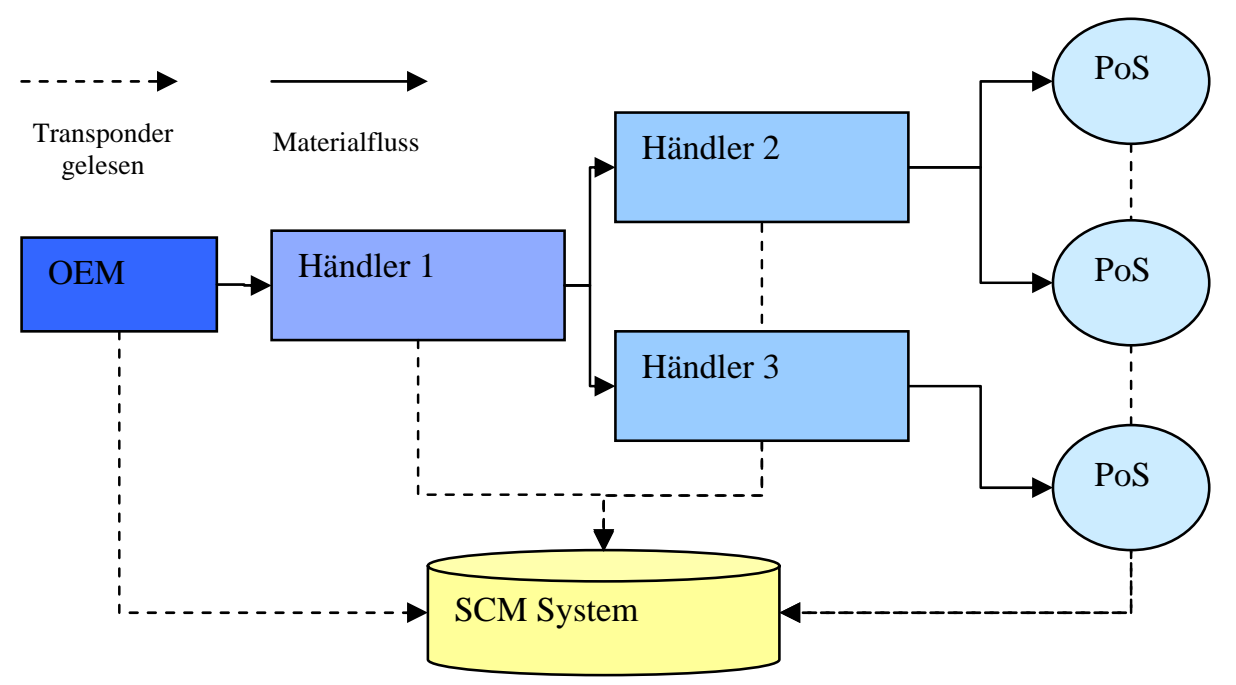

\begin{abstract}
Abbildung 1
Dieser Ansatz bietet enorme Vorteile, da alle Teile der Lieferkette transparent sind und Inkonsistenzen sofort aufgedeckt werden können. Verlässt das Produkt nun den Hersteller, wird dies in der zentralen Supply ChainSoftware automatisch durch das Auslesen des Transponders am Warenausgang mit einem Zeitstempel verbucht und der nachfolgende Händler bestätigt den Wareneingang ebenfalls automatisch in der Software. Dies setzt sich fort bis hin zum Point of Sale, und der Hersteller kann nachträglich überprüfen, ob die Produkte alle Teile der Lieferkette ordnungsgemäß durchlaufen haben. Treten nun Fälschungen im Handel auf, kann genau überprüft werden, an welcher Stelle innerhalb der Lieferkette Unregelmäßigkeiten aufgetreten sind.

Ist der Integrationsgrad der Supply Chain gering oder ist die Kostenaufteilung nicht gegeben, so kann ein dezentraler Ansatz gewählt werden, bei dem jeder Teil der Lieferkette über ein Schreib-/Lesegerät einen Zeitstempel und eine Signatur auf dem Transponder hinterlässt. Jeder Teilnehmer garantiert dann für die Integrität der Prozesse innerhalb seiner Unternehmensgrenzen gegenüber dem nachfolgenden Partner. Dies gewährleistet eine lückenlose Rückverfolgung, ohne dass unternehmensinterne Informationen preisgegeben werden müssen.
\end{abstract}

\title{
2. Fälschungssicherung durch verschlüsselte Transponder
}

Gemäß der Weltgesundheitsorganisation (WHO) sind weltweit 6\% aller Arzneimittel und Medikamente Fälschungen, dies entspricht einem Wert von 25 Milliarden Euro. Insbesondere in Afrika und in Asien liegen die Anteile jedoch deutlich höher, nach Schätzungen der WHO sind 50-60\% der in Afrika verkauften Medikamente gefälscht, Tendenz steigend. Während Fälschungen im Konsumgüterbereich jedoch meistens nur ärgerlich sind, können gefälschte Medikamente zu allergischen Reaktionen, gefährlichen Wechselwirkungen und sogar zum Tod der Patienten führen. Ein großer Teil der gefälschten Arzneien wird dabei über das Internet vertrieben.

Ist eine verstärkte Überwachung der Handelskette ein gutes Mittel, um das Einbringen von Fälschungen zu erschweren, so kann der Transponder auch als Echtheitssiegel dienen. Ähnlich der Wegfahrsperre im Auto wird ein Schlüssel auf dem RFID-Transponder hinterlegt, der eine eindeutige Authentifizierung erlaubt. Dabei übermittelt das Lesegerät eine Zufallszahl, die vom RFID-Transponder empfangen und zusammen mit den auf dem Transponder hinterlegten Daten (z. B. Hersteller, Name, Verfallsdatum, Wechselwirkungen, Allergieauslöser.....) durch den Transponder-Schlüssel codiert zurück übermittelt wird. Das Lesegerät entschlüsselt die Daten und erkennt anhand der Zufallszahl die Echtheit des Chips. Hier existieren bereits Transponder mit 1024 Bit Verschlüsselungen, die ohne Zuhilfenahme eines Supercomputers nicht zu "knacken“ sind; eine Verpackung mit fehlendem Transponder oder falschem Schlüssel ist somit schnell und einfach als Fälschung zu erkennen. Diese Methode würde es in Zukunft auch einem Endverbraucher ermöglichen, relevante Daten aus dem RFIDTransponder auszulesen und Produkte als echt zu identifizieren. Hier kann auch der Electronic Product Code (EPC) verwendet werden. Über den EPC kann ein Produkt eindeutig identifiziert werden, der Object Name Server (ONS) von EPC Global kann dieser EPC direkt zum Hersteller und zu entsprechenden Produktinformationen verlinken und diese Informationen dem Benutzer weiterleiten. 